

\subsection{1 .2010 \\ Lausanne}

Schweiz

\subsubsection{0}

Baden

Schweiz

09.-10.12.2010

Basel

Schweiz

.........

Wädenswil

Schweiz

\subsubsection{1}

Wädenswil

Schweiz

\section{Cours de base 2}

25. Schweizerische Jahrestagung für Phytotherapie: Phytotherapie -

Zukunft braucht Vergangenheit. Jubiläumstagung

4. ASA TCM-Kongress

Kurs 4. Arzt und Apotheker in der klinischen Forschung

Kurs 5. Phytotherapie bei Erkrankungen der Atemwege
Auskunft: SSPM, Madame Carmela Argenziano,

Avenue de Florimont 17, CH-1006 Lausanne,

Tel. +41216016920

sspm.phyto@gmail.com,www.sspm.org

Auskunft: SMGP, Kurs-Sekretariat, Irène Tinguely

Zürcher Hochschule für angewandte Wissenschaften

ZHAW Grüental

Postfach 335, CH-8820 Wädenswil

Tel. +41 44 78999-80, Fax -50

kurse-smgp.lsfm@zahw.ch, www.smgp.ch

Auskunft: www.congress-info.ch

Auskunft: SMGP, Kurs-Sekretariat, Irène Tinguely Zürcher Hochschule für angewandte Wissenschaften ZHAW Grüental

Postfach 335, CH-8820 Wädenswil

Tel. +41 44 78999-80, Fax -50

kurse-smgp.lsfm@zahw.ch, www.smgp.ch

Auskunft: SMGP, Kurs-Sekretariat, Irène Tinguely Zürcher Hochschule für angewandte Wissenschaften

ZHAW Grüental

Postfach 335, CH-8820 Wädenswil

Tel. +41 44 78999-80, Fax -50

kurse-smgp.lsfm@zahw.ch, www.smgp.ch 